\title{
On the application of Fubini's theorem in the integration of functions of two variables in a measure space
}

\author{
B.V. Akinremi*, B.N. Akintewe, K.S. Famuagun, U.S. Idiong \\ Adeyemi College of Education, Ondo, Nigeria \\ *Corresponding author E-mail: akinremibv@gmail.com
}

\begin{abstract}
We consider the integration of functions of two variables in a measure space. Some definitions, theorems and proves relating to measurable functions and measure space were considered by using Fubini's theorem. Application on the improvement of the Jensen's inequality with respect to the probability measure space is treated.
\end{abstract}

Keywords: Fubini's Theorem, Hermite-Hadamard inequality, Jensen's inequality, Measure, Product measure.

\section{Introduction}

In Mathematical analysis, integration of functions is of great importance. In the 1850's Bernhard Riemann adopted a new and different viewpoint of the calculus of integration by I. Newton and G. Leibniz. He separated the concept of integration from its companion, differentiation, and examined the motivating summation and limit process of finding areas by itself. He broadened the scope by considering all functions on an interval for which this process of integration could be defined: the class of 'integrable' functions. The viewpoint of Riemann led others to invent other integration theories, the most significant being Lebesgue's thoery of integration.

Measure theory was developed in successive stages during the late 19th- early 20th century by Emile Borel, Henri Lebesgue, Johann Radon among others. In measure integration theory, specifying a measure allows one to define integrals on spaces more general and it gives more theorem than its predecessor, the Riemann integral. Also, in classical analysis the problem of reducing double(or multiple) integrals to iterated integrals plays an important role. In integration theory, the key result along this line is the Fubini's theorem.

Fubini's theorem, named after Guido Fubini, is a result which gives conditions under which it is possible to compute a double integral using iterated integrals. As a consequence it allows the order of integration to be changed in iterated integrals.

In 1992 Dragomir and Ionescu [6] studied some aspects of convex functions and some interesting inequalities were obtained. Mitrinovic, Pecaric and Fink in [12] discussed classical and new inequalities in analysis like the Holder's, Minkowski's, Jensen's, Bernoullli's and Steffensen's inequalities in which they proved these inequalities with some generalizations. Dragomir and Ionescu [8] later in 1994 proved useful inequality which counterparts the Jensen's inequality for continuous functions. Rooin[14] proved some application on discrete function.

\section{Preliminary notes}

We briefly give some basic definitions of the concepts of measure, integration and product space which serves as background to this work.

Definiton 2.1.0[11] A system of set is a set whose elements are sets. A system of set $I$ is called a semiring if 
(i) $I$ contains the empty set.

(ii) $A \cap B \in I$ whenever $A \in I, B \in I$

(iii) if $A \in I$ and $A_{1} \subset A, A=\cup_{k=1}^{n} A_{k}$ such that $A_{K} \cap A_{l}=\phi \mathrm{k}=\mathrm{l}$ where $A_{1}$ is the first term.

Definiton 2.1.1[11] A set function is a function whose domain of definition is a class of sets. An extended real valued set function $\mathrm{m}$ defined on class of sets say $I_{m}$ with the following properties:

(i) the domain of definition $I_{m}$ of $\mathrm{m}$ is a semiring.

(ii) $\mathrm{m}$ is real and non-negative.

(iii) $\mathrm{m}$ is additive i.e if $P=\cup_{k=1}^{n} p_{k}$ then $m(P)=\sum_{k=1}^{n} m\left(p_{k}\right)$ where $p_{k}^{\prime} s$ are pairwise disjoint rectangles is called a measure.

Definition 2.1.2 Let $X$ be a set and $\mathcal{S}$ be a $\sigma$-algeba defined on $X$ and a measure $\mu$ defined on $X$, then the pair $(X, \mathcal{S})$ is called a measurable space and the triple $(X, \mathcal{S}, \mu)$ is called a measure space. e.g the probability measure space, complex measure space, projection-valued measure space. A measure space is called $\sigma$-finite if $X=\cup_{n} X_{n}$ where $m\left(X_{n}\right)<\infty$.

Definition 2.1.3[11] Let $f$ be a simple function then by the Lebesgue integral of $f$ over the set $A$ we mean

$$
\int_{A} f(x) d x=\sum_{n} y_{n} \mu\left(A_{n}\right) \quad A_{n}=\left\{x: x \in A, f(x)=y_{n}\right\}
$$

provided this series above is absolutely convergent. If the integral exist then we say $f$ is integrable with repect to the measure $\mu$ on the set $A$.

Definition 2.1.4[11] A measurable function $f$ is said to be integrable on a set $A$ if $\exists$ a sequence $\left\{f_{n}\right\}$ of integrable simple functions converging uniformly to $f$ on $A$. The $\operatorname{limit}_{n \rightarrow \infty} \lim _{A} f_{n}(x) d x$ is called the lebesgue integral of $f$ over set $A$.

$$
\lim _{n \rightarrow \infty} \int_{A} f_{n}(x) d x=\int_{A} f(x) d x
$$

Definition 2.1.5[15] Let $X$ and $Y$ be two sets, by product sets of $X$ and $Y$ we mean the set of all ordered pairs $(x, y)$ where $x \in X$ and $y \in Y$ and denoted by $X \times Y$. If $A \subset X$ and $B \subset Y$ then $A \times B \subset X \times Y$, we call set $A \times B$ a rectangle in $X \times Y$.

Definition 2.1.6[15] If $E \subset X \times Y$ such that $x \in X y \in Y$ we define

$$
E_{x}=\{y:(x, y) \in E\}, E^{y}=\{x:(x, y) \in E\}
$$

and $E_{x} \subset Y ; E^{y} \subset X$. Then all $E_{x}$ the x-section and $E^{y}$ the y-section.

Definition 2.1.7[15] Let $(X, S, \mu)$ and $(Y, T, \lambda)$ be measure spaces and if $Q \in S \times T$ we define

$$
(\mu \times \lambda)(Q)=\int_{X} \lambda\left(Q_{x}\right) d \mu(x)=\int_{Y} \mu\left(Q^{y}\right) d \lambda(y)
$$

$(\mu \times \lambda)$ is called the product of the measures $\mu$ and $\lambda$.

Definition 2.1.8[2] Let $(X, \mathcal{A}, \mu)$ and $(Y, \mathcal{B}, \lambda)$ be two probability measure spaces, by a weight function defined on $(X \times Y)$ we imply a product-measurable mapping $w: X \times Y \rightarrow[0, \infty)$ such that

$$
\begin{aligned}
& \int_{X} w(x, y) d \mu=1 \\
& \int_{Y} w(x, y) d \lambda=1
\end{aligned}
$$

\section{The Fubinni's theorem}

Let $f$ be a measurable function.

Theorem 3.1 If $E \in S \times T$, then $E_{x} \in T$ and $E^{y} \in S$ for every $x \in X$ and $y \in Y$. This theorem shows that $E_{x}$ and $E^{y}$ are measurable sets.

Theorem 3.2 Let $(X, S, \mu)$ and $(Y, T, \lambda)$ be $\sigma$-measure spaces. Supppose $Q \in S \times T$ and if

$$
\varphi(x)=\lambda\left(Q_{x}\right), \psi(y)=\mu\left(Q^{y}\right)
$$


for every $x \in X$ and $y \in Y$, then $\varphi$ is $S$ - measurable, $\psi$ is $T$ - measurable and

$$
\int_{X} \varphi d \mu=\int_{Y} \psi d \lambda
$$

where $\mu$ and $\lambda$ are positive measures on $S$ and $T$ respectively, $X=\cup_{n} X_{n}$ such that $\mu\left(X_{n}\right)<\infty$ and $Y=\cup_{m} Y_{m}$ such that $\lambda\left(Y_{m}\right)<\infty$. since by theorem 3.1

$$
\begin{array}{ll}
\lambda\left(Q_{x}\right) & =\int_{Y} \chi_{Q}(x, y) d \lambda(y) \quad(x \in X) \\
\mu\left(Q^{y}\right) & =\int_{X} \chi_{Q}(x, y) d \mu(x) \quad(y \in Y)
\end{array}
$$

with $(6) ;(7)$ can be expressed as

$$
\int_{X} d \mu(x) \int_{Y} \chi_{Q}(x, y) d \lambda(y)=\int_{Y} d \lambda(y) \int_{X} \chi_{Q}(x, y) d \mu(x)
$$

which is called an iterated integral.

Theorem 3.3 The Fubini's theorem[15]: Let $(X, S, \mu)$ and $(Y, T, \lambda)$ be a $\sigma-$ finite measure spaces and let $f$ be a $(S \times T)$ - measurable function on $X \times Y$

(a) If $0 \leq f \leq \infty$ and if

$$
\varphi(x)=\int_{Y} f_{x} d \lambda, \psi(y)=\int_{X} f^{y} d \mu \quad(x \in X, y \in Y)
$$

then $\varphi$ is $S-$ measurable, $\psi$ is $T$ - measurable,and

$$
\int_{X} \varphi d \mu=\int_{X \times Y} f d(\mu \times \lambda)=\int_{Y} \psi d \lambda
$$

(b) If $f$ is complex and if

$$
\varphi^{*}(x)=\int_{Y}|f|_{x} d \lambda \text { and } \int_{X} \varphi^{*} d \mu \leq \infty
$$

then $f \in L^{\prime}(\mu \times \lambda)$

(c) If $f \in L^{\prime}(\mu \times \lambda)$, then $f_{x} \in L^{\prime}(\lambda)$ for all $x \in X, f^{y} \in L^{\prime}(\mu)$ for almost all $y \in Y$, the functions $\varphi$ and $\psi$ defined by (7) a.e, are $L^{\prime}(\mu)$ and $L^{\prime}(\lambda)$,respectively and (10) holds.

Proof:

lemma 1:Let $\left\{f_{n}(x)\right\}$ be a sequence of non-negative monotonic increasing functions on a set $E$ and sequence that the sequence converges to $f(x)$ then

$$
\lim _{n \rightarrow \infty} \int_{E} f_{n}(x) d x=\int_{E} f(x) d x
$$

For (a) since $f_{x}$ and $f^{y}$ are $T$ - and $S$ - measurable the definition in equation (10) holds

$$
\varphi(x)=\int_{Y} f_{x} d \lambda, \quad \psi(y)=\int_{X} f^{y} d \mu \quad x \in X, y \in Y
$$

Suppose $Q \in S \times T$ and $f=\chi_{Q}$ by definition 2.1.7, equation (10) is exactly (7). Hence (a) holds $\forall$ non-negative simple $(S \times T)$-measurable function $s$. There is a sequence of such function $s_{n}$ such that $0 \leq s_{1} \leq s_{2} \leq \ldots \ldots$ and $s_{n}(x, y) \rightarrow f(x, y)$ at every of $(x, y) \in X \times Y$.But

$$
\begin{aligned}
\int_{X} \varphi(x) d \mu & =\int_{X \times Y} f d(\mu \times \lambda) \\
\Rightarrow \quad \int_{X} \varphi_{n}(x) d \mu & =\int_{X \times Y} s_{n} d(\mu \times \lambda) \quad(n=1,2, \ldots \ldots)
\end{aligned}
$$


by monotone convergence theorem [9] $\varphi_{n}(x) \rightarrow \varphi(x)$ for every $x \in X$ as $x \rightarrow \infty$. Applying this theorem on equation (12) we have

$$
\begin{gathered}
\lim _{n \rightarrow \infty} \int_{X} \varphi_{n} d \mu=\lim _{n \rightarrow \infty} \int_{X \times Y} s_{n} d(\mu \times \lambda) \\
\Rightarrow \int_{X} \varphi d \mu=\int_{X \times Y} f(x, y) d(\mu \times \lambda)
\end{gathered}
$$

hence the 1 st half of the inequality and associating $\psi$ with $s_{n}$ as

$$
\int_{Y} \psi d \lambda=\int_{X \times Y} f d(\mu \times \lambda)
$$

we have

$$
\int_{Y} \psi_{n} d \lambda=\int_{X \times Y} s_{n} d(\mu \times \lambda)
$$

by monotone convergence theorem we have

$$
\int_{Y} \psi d \lambda=\int_{X \times Y} f(x, y) d(\mu \times \lambda)
$$

hence (a) is proved and for (b) by (a) let $f=|f|$ then

$$
\varphi(x)=\int_{Y}|f|_{x} d \lambda \quad \psi(y)=\int_{X}|f|^{y} d \mu
$$

since $f$ is measurable so do $|f|[18]$ hence

$$
\varphi^{*}(x)=\int_{Y}|f|_{x} d \lambda \quad \text { and } \int_{X} \varphi^{*} d \mu<\infty
$$

$f \in L^{\prime}(\mu \times \lambda)$

For (c) we prove for real $L^{\prime}(\mu \times \lambda)$ then the complex case follows. If $f$ is real by (a) we have $f^{+}$and $f^{-}$.let $\varphi_{1}$ and $\varphi_{2}$ correspond to $f^{+}$and $f^{-}$as

$$
\begin{gathered}
\varphi(x)=\int_{X} f_{x} d \lambda \\
\Rightarrow \varphi_{1}(x)=\int_{X} f_{x}^{+} d \lambda \quad \varphi_{2}(x)=\int_{X} f_{x}^{-} d \lambda
\end{gathered}
$$

since $f \in L^{\prime}(\mu \times \lambda)$ and $f^{+} \leq|f|[18]$ and (a) holds for $f^{+}$then $\varphi_{1} \in L^{\prime}(\mu)$ also $f^{-} \leq|f|$ and $\varphi_{2} \in L^{\prime}(\mu)$ since

$$
f_{x}=\left(f^{+}\right)_{x}-\left(f^{-}\right)_{x}
$$

$f_{x}(x) \in L^{\prime}(\mu) \forall x$ for which $\varphi_{1}(x)<\infty$ and $\varphi_{2}(x)<\infty$ since $\varphi_{1}, \varphi_{2} \in L^{\prime}(\mu)$ which occurs for almost all $x$, and at such $x, \varphi(x)=\varphi_{1}(x)-\varphi_{2}(x)$ hence $\varphi \in L^{\prime}(\mu)$

$$
\begin{aligned}
\varphi_{1}(x)= & \int_{Y} f^{+} d \lambda, \quad \varphi_{2}(x)=\int_{Y} f^{-} d \lambda \quad(x \in X) \\
\varphi_{1}(x)-\varphi_{2}(x) & =\int_{Y} f^{+} d \lambda-\int_{Y} f^{-} d \lambda \\
\varphi(x) & =\int_{Y}\left(f^{+}-f^{-}\right) d \lambda \\
& =\int_{Y} f d \lambda
\end{aligned}
$$

hence $\varphi$ as defined in (7) a.e are in $L^{\prime}(\mu)$, also for $f$ real

$$
\psi_{1}(y)=\int_{X}\left(f^{+}\right)^{y} d \mu \quad \psi_{2}(y)=\int_{X}\left(f^{-}\right)^{y} d \mu
$$


since $f \in L^{\prime}(\mu \times \lambda)$ and $f^{+} \leq|f|$ and $f^{-} \leq|f|$ then (a) holds for $f^{+}$and $f^{-}$and $\psi_{1} \in L^{\prime}(\lambda), \quad \psi_{2} \in L^{\prime}(\lambda)$ since

$$
f^{y}=\left(f^{+}\right)^{y}-\left(f^{-}\right)^{y}
$$

we have $f^{y} \in L^{\prime}(\lambda) \forall y$ for which $\psi_{1}<\infty$ and $\psi_{2}<\infty$. since $\psi_{1}, \psi_{2} \in L^{\prime}(\lambda)$ which happens for almost all $y$ and at such $y, \psi(y)=\psi_{1}(y)-\psi_{2}(y)$. Hence $\psi \in L^{\prime}(\lambda)$. Now we have

$$
\begin{aligned}
\psi_{1}(y) & =\int_{Y}\left(f^{+}\right)^{y} d \mu, \quad \psi_{2}(y)=\int_{Y}\left(f^{-}\right)^{y} d \mu \quad y \in Y \\
\psi_{1}(y)-\psi_{2}(y) & =\int_{Y}\left(f^{+}\right)^{y} d \mu-\int_{Y}\left(f^{-}\right)^{y} d \mu \\
\psi(y) & =\int_{Y}\left[\left(f^{+}\right)^{y}-\left(f^{-}\right)^{y}\right] d \mu \quad \text { by } \quad(15) \\
& =\int_{Y} f^{y} d \mu
\end{aligned}
$$

Fubini's theorem asserts that if $f$ is $(\mathcal{S} \times \mathcal{T})$-measurable and if the iterated integral is finite then the two iterated integrals of $f$ are finite and equal.

\section{Application}

In this section, we will consider some applications as shown in the following examples

Example 4.1 We prove the Marcinkiewiez's theorem. Let F be a closed subset of a bounded open interval $(\mathrm{a}, \mathrm{b})$ and let $\delta(x)=\delta(x ; F)$ be the corresponding distance function then given $\lambda>0$, the interval

$$
M_{\lambda}=M_{\lambda}(x ; F)=\int_{a}^{b} \frac{\delta^{\lambda}(y)}{|x-y|^{1+\lambda}} d y
$$

is finite a.e in F.Moreover, $M_{\lambda} \in L(F)$ and

$$
\int_{F} M_{\lambda} d x \leq 2 \lambda^{-1}|G|
$$

where $G=(a, b)-F$.

To prove this we note the following:

(i) $\delta(x)=\delta(x ; F)=\inf \{|x-y|: y \in F\}$

(ii) $\delta(x)=o$ iff $x \in F$

sincce $\delta=0$ in $F$, integration in the integral defining $M_{\lambda}$ can be restricted to the set $G=(a, b)-F$ without changing $M_{\lambda}$ since the complement of $F$ is an open interval which is a union of $\cup_{k}\left(a_{k}, b_{k}\right)$ of disjoint open intervals, at most two of these interval can be infinite. Thus,

$$
\begin{aligned}
\int_{F} M_{\lambda}(x) d x & =\int_{F} \int_{a}^{b} \frac{\delta^{\lambda}(y)}{|x-y|^{1+\lambda}} d y d x \\
& =\int_{G} \delta^{\lambda}(y) \int_{F} \frac{d x}{|x-y|^{1+\lambda}} d y \quad \text { by Fubini's theorem }
\end{aligned}
$$

Suppose, we fix $y \in G$ and for any $y \in F$ we have $|x-y| \geq \delta(y)>0$, Thus,

$$
\begin{aligned}
& \leq \int_{G} \delta^{\lambda}(y)\left(\int_{|x-y| \geq \delta(y)} \frac{d x}{|x-y|^{1+\lambda}}\right) d y \\
& =\int_{G} \delta^{\lambda}(y)\left(2 \int_{\delta(y)}^{\infty} \frac{d t}{t^{1+\lambda}}\right) d y \\
& =2 \int_{G} \delta^{\lambda}(y)\left(\left[\frac{-\lambda^{-1}}{t^{\lambda}}\right]_{\delta(y)}^{\infty}\right) d y
\end{aligned}
$$




$$
\begin{aligned}
& =2 \int_{G} \delta^{\lambda}(y)\left(\frac{\lambda^{-1}}{\delta^{\lambda}(y)}\right) d y \\
& =2 \int_{G} \lambda^{-1} d y \\
& =2 \lambda^{-1}|G| \leq \infty
\end{aligned}
$$

Hence $M_{\lambda}(x)$ is finite and $M_{\lambda} \in L(F)$.

Remark: The finiteness of $M_{\lambda}(x)$ simply means $F$ is very Dense near $x$.

Example 4.2 We prove the Gaussian integral which is the basis for much of probability theory We prove that

$$
\int_{R^{n}} e^{-|x|^{2}} d x=\pi^{\frac{n}{2}}
$$

Proof: Suppose $n=1$

$$
\int_{R} e^{-|x|^{2}} d x=\pi^{\frac{1}{2}}
$$

then we have

$$
\left(\int_{R} e^{-|x|^{2}} d x\right)^{2}=\pi
$$

we show that the LHS equals the RHS

$$
\begin{aligned}
\left(\int_{R} e^{-|x|^{2}} d x\right)^{2} & =\left(\int_{-\infty}^{\infty} e^{-|x|^{2}} d x\right)^{2} \\
& =\left(2 \int_{0}^{\infty} e^{-|x|^{2}} d x\right)^{2} \\
& =4 \int_{0}^{\infty} \int_{0}^{\infty} e^{-|x|^{2}} e^{-|y|^{2}} d x d y \\
& =4 \int_{0}^{\infty} \int_{0}^{\infty} e^{-\left(x^{2}+y^{2}\right)} d x d y
\end{aligned}
$$

let $r^{2}=x^{2}+y^{2} d x d y=r d r d \theta, x=r \cos \theta, y=r \sin \theta x=0$ to $\infty$ implies $r=0$ to $\infty$ and $y=0$ to $\infty$ implies $\theta=0$ to $\frac{\pi}{2}$

$$
\begin{aligned}
4 \int_{0}^{\infty} \int_{0}^{\infty} e^{-\left(x^{2}+y^{2}\right)} d x d y & =4 \int_{0}^{\infty} \int_{0}^{\frac{\pi}{2}} e^{-r^{2}} r d r d \theta \\
& =4 \int_{0}^{\infty} \int_{0}^{\frac{\pi}{2}} \frac{e^{-t}}{2} d t d \theta \\
& =2 \int_{0}^{\infty} \int_{0}^{\frac{\pi}{2}} e^{-t} d t d \theta \\
& =2 \int_{0}^{\frac{\pi}{2}}\left[e^{-t}\right]_{0}^{\infty} d \theta \\
& =2 \int_{0}^{\frac{\pi}{2}} d \theta=\pi
\end{aligned}
$$

hence $\left(\int_{R} e^{-|x|^{2}} d x\right)^{2}=\pi$ suppose $n \geq 1,\left(\int_{R^{n}} e^{-|x|^{2}} d x\right)^{2}$ let $e^{-|x|^{2}}=e^{-x_{1}^{2}} e^{-x_{2}^{2}} e^{-x_{3}^{2}} \cdots e^{-x_{n}^{2}}$ then we have

$$
\begin{gathered}
\int_{R^{n}} e^{-|x|^{2}} d x=\int_{R^{1}} \int_{R^{2}} \cdots \int_{R^{n}} e^{-x_{1}^{2}} e^{-x_{2}^{2}} \cdots e^{-x_{n}^{2}} d x_{1} d x_{2} \cdots d x_{n} \\
\left(\int_{R^{n}} e^{-|x|^{2}} d x\right)^{2}=\left(\int_{R_{1}} \int_{R_{2}} \cdots \int_{R_{n}} e^{-x_{1}^{2}} e^{-x_{2}^{2}} \cdots e^{-x_{n}^{2}} d x_{1} d x_{2} \cdots d x_{n}\right)^{2}
\end{gathered}
$$




$$
\begin{aligned}
& =\int_{R_{1}} \int_{R_{1}} \int_{R_{2}} \int_{R_{2}} \cdots \int_{R_{n}} \int_{R_{n}} e^{-x_{1}^{2}} e^{-y_{1}^{2}} e^{-x_{2}^{2}} e^{-y_{2}^{2}} \cdots e^{-x_{n}^{2}} e^{-y_{n}^{2}} d x_{1} d y_{1} d x_{2} d y_{2} \cdots d x_{n} d y_{n} \\
& =\int_{R_{1}} \int_{R_{1}} \int_{R_{2}} \int_{R_{2}} \cdots \int_{R_{n}} \int_{R_{n}} e^{-\left(x_{1}^{2}+y_{1}^{2}\right)} e^{-\left(x_{2}^{2}+y_{2}^{2}\right)} \cdots e^{-\left(x_{n}^{2}+y_{n}^{2}\right)} d x_{1} d y_{1} d x_{2} d y_{2} \cdots d x_{n} d y_{n} \\
& =\int_{R_{1}} \int_{R_{1}} \cdots \int_{R_{n-1}} \int_{R_{n-1}}\left(\int_{R_{n}} \int_{R_{n}} e^{-\left(x_{1}^{2}+y_{1}^{2}\right)} e^{-\left(x_{2}^{2}+y_{2}^{2}\right)} \cdots e^{-\left(x_{n}^{2}+y_{n}^{2}\right)} d x_{n} d y_{n}\right) d x_{n-1} d y_{n-1} \cdots d x_{1} d y_{1} \\
& =\int_{R_{1}} \int_{R_{1}} \cdots \int_{R_{n-1}} \int_{R_{n-1}}\left(\pi e^{-\left(x_{1}^{2}+y_{1}^{2}\right)} e^{-\left(x_{2}^{2}+y_{2}^{2}\right)} \cdots e^{-\left(x_{n-1}^{2}+y_{n-1}^{2}\right)}\right) d x_{n-1} d y_{n-1} \cdots d x_{1} d y_{1} \\
& =\int_{R_{1}} \int_{R_{1}} \cdots \int_{R_{n-2}} \int_{R_{n-2}}\left(\pi^{2} e^{-\left(x_{1}^{2}+y_{1}^{2}\right)} e^{-\left(x_{2}^{2}+y_{2}^{2}\right)} \cdots e^{-\left(x_{n-2}^{2}+y_{n-2}^{2}\right)}\right) d x_{n-2} d y_{n-2} \cdots d x_{2} d y_{2} d x_{1} d y_{1} \\
& =\cdots \cdots \\
& =\int_{R_{1}} \int_{R_{1}} \int_{R_{2}} \int_{R_{2}} \pi^{n-2} e^{-\left(x_{1}^{2}+y_{1}^{2}\right)} e^{-\left(x_{2}^{2}+y_{2}^{2}\right)} d x_{2} d y_{2} d x_{1} d y_{1} \\
& =\int_{R_{1}} \int_{R_{1}} \pi^{n-1} e^{-\left(x_{1}^{2}+y_{1}^{2}\right)} d x_{1} d y_{1} \\
& =\pi^{n-1}(\pi)
\end{aligned}
$$

therefore

$$
\begin{aligned}
\left(\int_{R^{n}} e^{-|x|^{2}} d x\right)^{2} & =\pi^{n} \\
\int_{R^{n}} e^{-|x|^{2}} d x & =\pi^{\frac{n}{2}}
\end{aligned}
$$

Theorem 4.3 Let $(X, \mathcal{A}, \mu)$ and $(Y, \mathcal{B}, \lambda)$ be two probability spaces and $w: X \times Y \rightarrow[0,1)$ be a weight function on $X \times Y$. If I is an interval of the real line $f(x) \in L^{\prime}(\mu), f(x) \in I \forall x \in X$ and $\varphi$ is a real convex function on $I$ then

$$
\int_{Y} \varphi \int_{X} f(x) w(x, y) d \mu d \lambda
$$

and

$$
\varphi \int_{X} f(x) d \mu \leq \int_{Y} \varphi \int_{X} f(x) w(x, y) d \mu d \lambda \leq \int_{X}(\varphi \cdot f)(x) d \mu
$$

Let $X=\{0,1\}$ with $\mathcal{A}=2^{X}$ and $\mu\{0\}=\mu\{1\}=\frac{1}{2}, Y=[0,1]$ with Lebesgue measure $\lambda$. If $\varphi$ is a convex function on a closed interval $I$ such that $I=[\mathrm{a}, \mathrm{b}]$ then

$$
\varphi\left(\frac{a+b}{2}\right) \leq \frac{1}{(b-a)} \int_{a}^{b} \varphi(t) d t \leq \frac{\varphi(a)+\varphi(b)}{2}
$$

\section{Proof:}

$X=\{0,1\} \mu\{0\}=\mu\{1\}=\frac{1}{2}$ and $I=[\mathrm{a}, \mathrm{b}]$ and by $(10)$

$$
\varphi \int_{X} f(x) d \mu \leq \int_{Y} \varphi \int_{X} f(x) w(x, y) d \mu d \lambda \leq \int_{X}(\varphi \cdot f)(x) d \mu
$$

taking $w: X \times Y \rightarrow[0, \infty)$ as a weight function defined $w(x, y)=|2(x-1)+2 y|$ then we have $w(0, y)=2(1-y)$ and $w(1, y)=2 y$ for $y \in[0,1]$, by theorem $4.2 f(x) \in I$, let $f(0)=a$ and $f(1)=b$ since $I=[a, b]$ then

$$
\begin{aligned}
\varphi \int_{X} f d \mu & =\varphi\left(\int_{x=0} f(x) d x+\int_{x=1} f(x) d x\right) \\
& =\varphi\left(\frac{f(0)}{2}+\frac{f(1)}{2}\right) \\
& =\varphi\left(\frac{a+b}{2}\right)
\end{aligned}
$$




$$
\begin{aligned}
\int_{Y} \varphi \int_{X} f(x) w(x, y) d \mu d \lambda & =\int_{Y} \varphi\left(\int_{x=0} f(x) w(x, y) d x+\int_{x=1} f(x) w(x, y) d x\right) d \lambda \\
& =\int_{Y} \varphi\left(\int f(x) w(0, y) d x+\int f(x) w(1, y) d x\right) d y \\
& =\int_{Y} \varphi\left(a \frac{2(1-y)}{2}+b \frac{2 y}{2}\right) d y \\
& =\int_{Y} \varphi(a(1-y)+b y) d y
\end{aligned}
$$

let $t=a(1-y)+b y$ then $\frac{d t}{d y}=b-a$ and $d y=\frac{d t}{b-a}$

$$
\begin{aligned}
& =\int_{a}^{b} \frac{\varphi(t)}{b-a} d t \\
& =\frac{1}{b-a} \int_{a}^{b} \varphi(t) d t
\end{aligned}
$$

and

$$
\begin{aligned}
\int_{X}(\varphi \cdot f) d \mu & =\int_{x=0}(\varphi f(x))+\int_{x=1}(\varphi \cdot f(x)) d x \\
& =\frac{\varphi(a)}{2}+\frac{\varphi(b)}{2} \\
& =\frac{\varphi(a)+\varphi(b)}{2}
\end{aligned}
$$

hence the result follows from (18), (19) and (20).

Remark: If $\varphi$ is a real convex function on a closed interval $[a, b]$, then we have the Hermite-Hadamard inequality.

\section{Conclusion}

In this work we discussed the concepts of measure space and integration of functions of two variables, where definitions, some theorems and proofs were treated. We considered integrating functions of two variables in measure space using Fubini's theorem with some applications. Futhermore, we were able to use the generalized Jensen's inequality to prove the Hermite-Hadamard inequality. In this study, we conclude that the Fubini's theorem is of great importance in the integration of function of two variables in measure space.

\section{References}

[1] T.M. Apostol, Mathematical Analysis,2nd Edition,Addison-Wesley Publishing Company 1974.

[2] D. Bainor, P. Simeonor, Integral Inequalities and Application, Academic Publishers, Drodrecht 1992.

[3] I. Budimir, S.S Dragomir, J. Pecaric, Further Reverse results for Jensen's Discrete Inequality and Application in Information theory. Vol 2(1) article 2001.

[4] S. Chandra, S.M Shah, Introduction to Real Variable Theory, Prentice-Hall of Intia limited, New Dehli 1987.

[5] G. Choguct, Lectures on Analysis 'Integration and Topological Vector spaces' Vol 1 N.A Benjamin,Inc 1976.

[6] S.S. Dragomir, N.M. Ionescu, Some remarks on Convex functions, Revue d'analysis numerique et de theorie de l'approximation 21,1992 pp $31-36$.

[7] S.S. Dragomir, A refinement of Jensen's inequality with application for f-Divergence measure,(http://rgmia.vu.edu.a/dragomir).

[8] S.S. Dragomir, N.M. Ionescu, Some converse of Jensen's inequality and application; Anal. Num. Theor. Approx. (Chij - Napoca) 23,1994 Pp 71 -78.

[9] P.R. Halmos, Measure Theory, Springer-Verlag, New York 1974. 
[10] E. Hewitt, K. Stromberg, Real and Abstract Analysis, Springer-Verlag, New York 1965.

[11] A.N. Kolmogorov, S.V. Fomin, Introductory Real Analysis, Dover Publication,Inc. 1975

[12] D.E. Mitrinoric, J.E Pecaric, A.M. Fink, Classical and New Inequalities in Analysis, khiwer Academic Dordrecht 1993.

[13] C.C. Pugh, Real Mathematical Analysis, Springer-Verlag, New York 1940.

[14] J. Rooin, Some aspects of convex functions and their Application, Journal of Inequality in Pure and Applied Mathematics,vol 2(1)article 42001.

[15] W. Rudin, Real and Complex Analysis, 3rd Edition, McGraw-Hill, New York. 1974.

[16] W. Rudin, Principles of Mathematical Analysis,3rd Ed McGraw-Hill,Inc.New York. 1976.

[17] S. Sternberg, Theory functions of a Real Variable, lecture note, MAY 102005.

[18] M.P. Spiegel, Schaum's Outline of theory of problems of real variables, Lebesgue Measure and Integration with Application to Fourier Series 1968.

[19] A.J. Weir, Lebesgue Integration and Measure, Cambridge University press 1973.

[20] R.L. Wheeden, A. Zygmund, Measure and Integral:An introduction to Real Analysis, Marcel Dekker,Inc. New York. 1977. 